\title{
Fatores influentes na desistência de idosos em um programa de exercício físico
}

\author{
Adilson Sant'Ana Cardoso* \\ Lucélia Justino Borges** \\ Giovana Zarpellon Mazo*** \\ Tânia Bertoldo Benedetti**** \\ Anna Paula Kuhnen ${ }^{* * * * *}$
}

\begin{abstract}
Resumo: O presente estudo investigou os fatores que influenciam na adesão e desistência em um programa de exercício físico para idosos implantado nas Unidades Locais de Saúde (ULS) de Florianópolis, SC. Para isso, foram entrevistados 13 idosos, com idade média de 71,08 $(D P=5,75)$ anos, por meio de uma entrevista semiestruturada aplicada via telefone. Concluiu-se que o principal motivo para adesão ao programa é o aspecto relacionamento (convite de amigos e/ou familiares) e dentre os motivos de desistência estão causas pessoais (problemas de saúde do cônjuge, morte do mesmo ou demais familiares) foram os mais freqüentes.
\end{abstract}

Palavras-chave: Exercício. Adesão. Desistência do paciente. Idoso.

\footnotetext{
* Mestrando em Ciências do Movimento Humano, Laboratório de Gerontologia (LAGER), Centro de Ciências da Saúde e do Esporte (CEFID), Universidade do Estado de Santa Catarina (UDESC) Florianópolis, SC, Brasil. E-mail: cardosoas@gmail.com.

" Mestranda em Educação Física, Núcleo de Cineantropometria \& Desempenho Humano (NuCIDH), Universidade Federal de Santa Catarina (UFSC).Departamento de Educação Física. Florianópolis, SC, Brasil. E-mail: luceliajb@yahoo.com.br

"* Doutora em Educação Física, Laboratório de Gerontologia (LAGER), Centro de Ciências da Saúde e do Esporte (CEFID), Universidade do Estado de Santa Catarina (UDESC). Florianópolis, SC, Brasil. E-mail: d2gzm@udesc.br

${ }_{* * *}$ Doutora em Enfermagem, Núcleo de Cineantropometria \& Desempenho Humano (NuCIDH) Universidade Federal de Santa Catarina (UFSC), Departamento de Educação Física. Florianópolis, SC, Brasil. E-mail: trbbcds@yatech.net

**** Mestranda em Educação Física. Núcleo de Cineantropometria \& Desempenho Humano (NuCIDH), Universidade Federal de Santa Catarina (UFSC). Palhoça, SC,Brasil. E-mail: aninhakuhnen@yahoo.com.br .
} 


\section{INTRODUÇÃo}

O Brasil passa por um processo de envelhecimento populacional, como em todos os locais do mundo. Contudo, o aumento na expectativa média de vida do brasileiro não foi devidamente acompanhado por melhorias e incrementos significativos na qualidade de vida da população, resultando em uma expectativa de vida livre de incapacidades de apenas 54 anos (IBGE, 2000). Nesse ponto, assumir um estilo de vida ativo é fundamental para a manutenção da qualidade de vida do indivíduo (MAZO, 2003).

$\mathrm{O}$ envolvimento regular na prática de atividades e exercícios físicos pode retardar o declínio normal relacionado à idade na função dos diferentes sistemas. Pode também, controlar e prevenir doenças crônico-degenerativas (cardiovasculares, diabetes, câncer, hipertensão, etc.) que podem levar a debilidades (SHEPHARD, 2003; BIRD; TARPENNING; MARINO, 2005). Warburton, Nicol e Bredin (2006), indicam que há uma relação linear entre atividade física e o estado de saúde, sendo que o aumento nos níveis de atividade física pode levar a incrementos e melhorias no estado de saúde do indivíduo.

Porém, apesar da literatura evidenciar a importância e os benefícios do exercício físico para um envelhecimento ativo e saudável, os esforços de autoridades e pesquisadores parecem ser insuficientes para sensibilizar a maior parte da população a participar de maneira regular desses programas. De acordo com pesquisa recente nas capitais brasileiras, a inatividade física atinge grande parte da população idosa, representado nos $50,3 \%$ das mulheres e $65,4 \%$ dos homens acima dos 65 anos sem atividade. (BRASIL..., 2007).

Nesse contexto, detectam-se altas taxas de inatividade e conseqüentemente baixa porcentagem de idosos inseridos em programas de atividade física sistematizada. A literatura aponta a existência de fatores que influenciam essa prática tanto de forma positiva, quanto negativa. Os fatores que facilitam, oportunizam ou viabilizam essa prática, são chamados "facilitadores", e os que atrapalham ou dificultam, são chamados de "barreiras". As barreiras 
podem ser: internas: aquelas que dizem respeito ao pensamento individual, ao sentimento do idoso, à forma como fazem a leitura de um fenômeno ou fato; e externas: aquelas que se referem ao ambiente, a situações e à sociedade em geral (MICHELI, 2007).

Portanto verifica-se que existem vários fatores que auxiliam tanto a inserção quanto a aderência do idoso em programas de exercícios físicos, e isso passa por aspectos da educação, do âmbito familiar e social, da área da saúde e da economia, da quebra de vários paradigmas, enfim, dos aspectos que permeiam a qualidade de vida da população (MICHELI, 2007).

Diante disso, faz-se necessário não só reforçar os fatores facilitadores para a inserção do idoso no programa de exercício físico, mas também, conhecer e buscar diminuir as barreiras que têm favorecido a desistência desses idosos no programa. Mazo (2003) indica que a associação de barreiras com a atividade física é altamente pertinente para as intervenções, uma vez que o reconhecimento destas pode guiar os programas e suas ações, de forma a definir metodologias e estratégias mais eficientes. Cohen-Mansfield (et al., 2004) ressalta ainda que os programas devem levar em conta as necessidades e desejos dos subgrupos, bem como, explorar o significado que os idosos conferem aos programas para adaptar seu design para uma intervenção mais efetiva.

Assim, o presente estudo teve por objetivo investigar os fatores influentes na desistência de idosos em um programa de exercício físico implantado nas Unidades Locais de Saúde (ULS) de Florianópolis no Estado de Santa Catarina.

\section{Metodologia}

Esta pesquisa foi encaminhada e aprovada pelo Comitê de Ética de Seres Humanos da Universidade Federal de Santa Catarina, projeto $\mathrm{n}^{\mathrm{o}}$ 011/06 da data de 06 de março de 2006. Todos os idosos foram informados sobre a pesquisa e assinaram o Termo de

Movimento, Porto Alegre, v. 14, n. 01, p. 225-239, janeiro/abril de 2008. 
Consentimento Livre e Esclarecido - TCLE em duas vias, ficando uma de posse dos idosos e outra dos pesquisadores.

\subsection{População e Amostra}

A população do estudo foi composta por 16 idosos desistentes do programa "Idoso Ativo", entre os meses de julho e dezembro de 2006.

O programa de exercício físico teve seu início em junho de 2006, por meio de aulas de ginástica que eram realizadas três vezes/ semana, com duração de 60 minutos/sessão, enfatizando as diferentes qualidades físicas, principalmente a força, equilíbrio, flexibilidade, coordenação e resistência aeróbica; além de exercícios específicos para reeducação postural, fortalecimento da musculatura pélvica e descontração muscular por meio de relaxamento.

Os critérios de inclusão na amostra foram: ter mais de 15 faltas consecutivas ( $25 \%$ do total de aulas dadas) entre os meses de julho e dezembro de 2006 - critério adotado pelos pesquisadores para caracterizar a desistência do programa - não ter retornado às atividades do programa até a realização da coleta de dados do presente estudo; concordar e ter disponibilidade para participar da pesquisa, respondendo individualmente à entrevista via telefone.

Atenderam aos critérios de inclusão 16 idosos, com idade entre 60 a 82 anos. Três idosos foram excluídos, sendo um por problemas mentais e dois por não terem sido localizados, o que impossibilitou a realização da entrevista. Dessa forma, a amostra do presente estudo foi composta por 13 idosos (11 do sexo feminino e dois do sexo masculino), com idade média de 71,08 anos $(\mathrm{DP}=5,75)$, que responderam a entrevista.

\subsection{Instrumentos}

Para averiguar os fatores contribuintes para a desistência do programa "Idoso Ativo" foi elaborada uma entrevista semiestruturada, com oito perguntas relacionadas às temáticas abordadas.

Movimento, Porto Alegre, v. 14, n. 01, p. 225-239, janeiro/abril de 2008. 
Inicialmente as questões abordadas na entrevista referiam-se à lembrança da data de entrada, bem como os motivos que levaram à sua participação no programa, para deste ponto em diante serem abordadas as questões relacionadas à desistência e à possibilidade de retorno ao programa. Estas últimas foram avaliadas por meio das seguintes questões: Qual(is) o(s) motivo(s) que levou (levaram) a sair da ginástica?; O que o Sr.(a) achava das aulas de ginástica (professora, aula, material, músicas, horário, local)?; O Sr.(a) sentia-se bem quando participava da ginástica?; O Sr.(a) gostaria de voltar a participar da ginástica?; O que teria que acontecer para possibilitar a sua volta à ginástica?Acha que isso é possível?

\subsection{Coleta de Dados}

Os dados foram coletados por meio de entrevista individual via telefone, realizada no mês de maio de 2007. Inicialmente os pesquisadores se identificavam, em seguida apresentavam o objetivo do contato. As entrevistas duraram entre seis e 13 minutos, sendo o tempo médio de entrevista de 9 min53 s.

\subsection{Análise de Dados}

Os dados coletados foram organizados armazenados e tratados com auxílio do programa Microsoft Excel. A análise adotada para o tratamento dos dados foi a estatística descritiva (percentagem).

\section{RESULTADOS E DISCUSSÃo}

Quanto ao perfil sócio-demográfico dos idosos, detectou-se que $84,6 \%$ são do sexo feminino, sendo que a idade média obtida foi de 71,08 anos ( $\mathrm{DP}=5,75)$. Em relação ao estado civil, 69,2\% são casados; $23,1 \%$ viúvos e $7,7 \%$ são divorciados ou separados. Quanto à escolaridade $35,2 \%$ tem de um a três anos de estudo formal, sendo o mesmo percentual encontrado para aqueles que têm entre quatro a oito anos; $15,4 \%$ têm mais de oito anos de estudo e o percentual de analfabetos encontrado foi de $7,7 \%$.

Movimento, Porto Alegre, v. 14, n. 01, p. 225-239, janeiro/abril de 2008. 
O tempo de permanência no programa foi de $84,6 \%$ dos idosos entrevistados, sendo que, variou de um a seis meses, e a maior freqüência observada na categoria de três a cinco meses $(38,5 \%)$, seguido da categoria até dois meses $(30,8 \%)$. Contudo apenas $69,2 \%$ afirmaram lembrar a data de inserção no programa, o que nos faz questionar e repensar sobre os dados obtidos a respeito do tempo de permanência. Como o idoso pode se lembrar do tempo que permaneceu no programa, se não lembra a data de seu ingresso?

Dentre os motivos que levaram à participação no programa, $\mathrm{o}$ relacionamento foi o que apresentou maior freqüência (Gráfico 1), salientando que o convite de outras pessoas (amigos, vizinhos, colegas de grupo de convivência e família), pode ser efetivo para engajamento dessa população em programas de exercício físico.

O resultado obtido confronta-se com resultados apresentados por Freitas et al. (2007), em um estudo recente que avaliou os motivos de aderência e permanência de praticantes de dois programas de exercício físico em Recife, onde foram entrevistados 120 idosos com mais de 60 anos que haviam permanecido por mais de seis meses nos programas. Nesse estudo, a influência de familiares e amigos foi apontada por $28,3 \%$ como "sem importância", bem como $23,3 \%$ dos entrevistados citaram a companhia de colegas com a mesma classificação. No mesmo estudo, os aspectos relacionados à melhora da saúde, desempenho físico e adoção de um estilo de vida saudável, dentre outros, foram apontados como causas de aderência "importantíssimas" pelos idosos entrevistados (FREITAS et al., 2007). Cohen-Mansfield et al. (2004) analisaram 324 idosos com idades entre 74 e 85 anos, concluindo que, para quase $70 \%$ dos participantes, causas como: aconselhamento médico para a prática de exercício físico; ser monitorado por um profissional da saúde; a qualidade do instrutor e a avaliação dos efeitos do exercício por um profissional eram importantes ou muito importantes para os idosos se engajarem no programa. Os autores citam ainda outras causas como muito importantes: fácil acesso ao local das aulas (preferencialmente perto de casa), tipo de exercício realizado, custo, questões sociais (grupo sendo da mesma idade).

Movimento, Porto Alegre, v. 14, n. 01, p. 225-239, janeiro/abril de 2008. 
No presente estudo observou-se que as respostas pertinentes à aderência ao programa deveu-se: $23,5 \%$ das melhora da saúde como causa principal, e também, $23,5 \%$ indicaram a orientação médica.

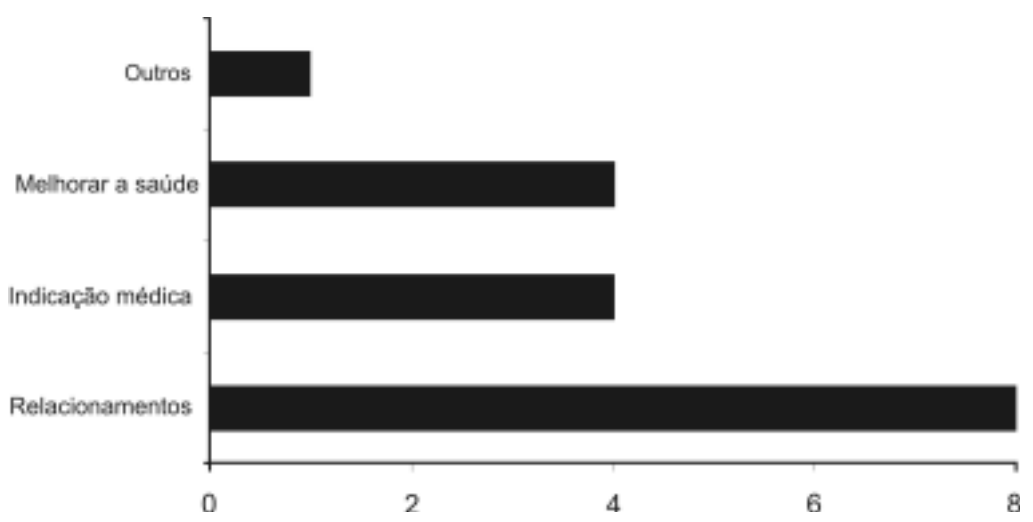

Gráfico 1: Aspectos que levaram os idosos a iniciarem a participação no programa de ginástica. Para esses aspectos uma ou mais opções foram referendadas e por isso existe discrepância quanto ao total das freqüências e a amostragem.

A motivação pode ser definida como alguma força interior, impulso ou uma intenção, que leva uma pessoa a fazer algo ou agir de certa forma, ou seja, como a causa de um comportamento (MAGILL, 1984). Schutzer e Graves (2004), em um estudo de revisão, apontam como aspectos motivacionais para o exercício físico em idosos: a auto-eficácia, a recepção de alertas informativos, a música no ambiente e questões demográficas. Mazo (2003) ressalta ainda fatores que podem interferir na prática de atividades e exercícios físicos: a história, a socialização, a discriminação da idade, fatores sociais e ambientais, benefícios percebidos e expectativas dos resultados, bem como atitudes e conhecimento.

A auto-eficácia - definida como uma crença pessoal do indivíduo em seu sucesso ao realizar alguma tarefa (BANDURA, 1977) - é consistentemente identificada como um fator para a adesão e manutenção da prática do exercício físico em diversas populações, inclusive em idosos. Receber contatos informativos via telefone,

Movimento, Porto Alegre, v. 14, n. 01, p. 225-239, janeiro/abril de 2008. 
correio ou pessoalmente também parecem serem fatores motivadores. Outro facilitador da prática de exercício é ter presente no ambiente uma música apropriada, pois parece diminuir a percepção de dificuldade, monotonia e desconfortos associados ao exercício físico. As características demográficas mais associadas à motivação para manter-se em programas de exercícios físicos relacionam-se a indivíduos mais aptos no início do programa, com menos doenças crônicas e dores, não fumantes, dentre outras (SCHUTZER; GRAVES, 2004).

Cabe ressaltar, que a orientação médica para a prática de exercício físico é apontada como um fator chave para que o idoso assuma um comportamento ativo (COHEN-MANSFIELD et al., 2004), em virtude do respeito que os idosos têm acerca dos conselhos dados pelo seu médico (SCHUTZER; GRAVES, 2004). Um estudo realizado na cidade de Baltimore - EUA, com 2507 idosos beneficiários do sistema de saúde por quatro anos, indicou que dos 301 idosos que iniciaram um estilo de vida ativo, durante o período do estudo, $40 \%$ apontaram a influência do médico como muito importante (BURTON; SHAPIRO; GERMAN, 1999). Também Calfas et al. (1996) indicaram que o aconselhamento do médico para a adoção de um estilo de vida ativo é eficiente para indivíduos previamente sedentários.

Contudo, a orientação médica pode também ser considerada uma barreira para a adoção de um estilo de vida ativo, visto que, como citam Schutzer e Graves (2004), uma vez que estes podem não aconselhar regular e corretamente os idosos para a prática de atividades e exercícios físicos.

Um fator que pode contribuir para a não adoção ou não manutenção de um estilo de vida ativo pelos idosos, e se acredita relevante, refere-se à falta de conhecimento, ou entendimento, acerca da relação entre exercício físico moderado e saúde (MAZO, 2003; SCHUTZER; GRAVES, 2004). Para Schutzer e Graves (2004), esta é uma barreira importante, pois muitos idosos viveram em épocas em que o exercício físico não estava relacionado à saúde, não sendo valorizado nem considerado necessário, tendendo assim

Movimento, Porto Alegre, v. 14, n. 01, p. 225-239, janeiro/abril de 2008. 
a perceberem o exercício por um viés recreacional (CHAO et al., 2000).

Todavia, a relação entre atividade, exercício físico e saúde já está confirmada por diversos estudos (SHEPHARD, 2003; BIRD; TARPENNING; MARINO, 2005; WARBURTON; NICOL; BREDIN, 2006), cabendo aos profissionais que lidam com essa população a transmissão e o correto aconselhamento. Infelizmente, como citam Chao et al. (2000), muitos profissionais responsáveis pela saúde do idoso freqüentemente não informam com clareza a respeito dos exercícios físicos. Acostumaram-se a aconselhar aos idosos que sejam mais ativos, mas não oferecem condutas específicas e maiores esclarecimentos. Acredita-se que uma das possíveis causas para isso é a não indicação de um profissional de Educação Física, que tem dentre as suas especificidades de intervenção profissional, a prescrição, orientação e planejamento do programa de exercício físico.

Outras barreiras apresentadas pelos idosos para a prática das atividades e exercícios físicos são freqüentemente associadas a um estado de saúde debilitado, ao ambiente físico onde ocorre a prática da atividade (como calçadas, parques, centros recreativos, e outros fatores facilitadores presentes, bem como vizinhanças tranqüilas) e também, apesar de apresentar fraca relação, as influências da atividade física na infância e adolescência do idoso (SCHUTZER; GRAVES, 2004). Os problemas de saúde são apontados por diversos autores (SANTARIANO et al., 2000; DERGANCE et al., 2003; MAZO, 2003) como sendo uma das barreiras mais freqüentes para a prática de atividades ou exercícios físicos nos idosos. Em um estudo com 199 idosos, O’Neil e Reid (1996) indicaram que 87\% dos entrevistados apresentavam pelo menos uma barreira para a prática de exercício físico.

Quanto aos motivos de desistência observados no presente estudo, os principais motivos destacados pelos idosos foram agrupados em três categorias principais, sendo elas: 1) pessoais: problemas de saúde ou morte do cônjuge ou de outros familiares; 2) saúde: cirurgias, tratamentos de doenças como labirintite e hérnia 
de estômago; 3) exercício físico: dores articulares sentidas após as aulas realizadas com exercícios realizados nas posições sentada e deitada. Esses resultados podem ser visualizados no Gráfico 2.

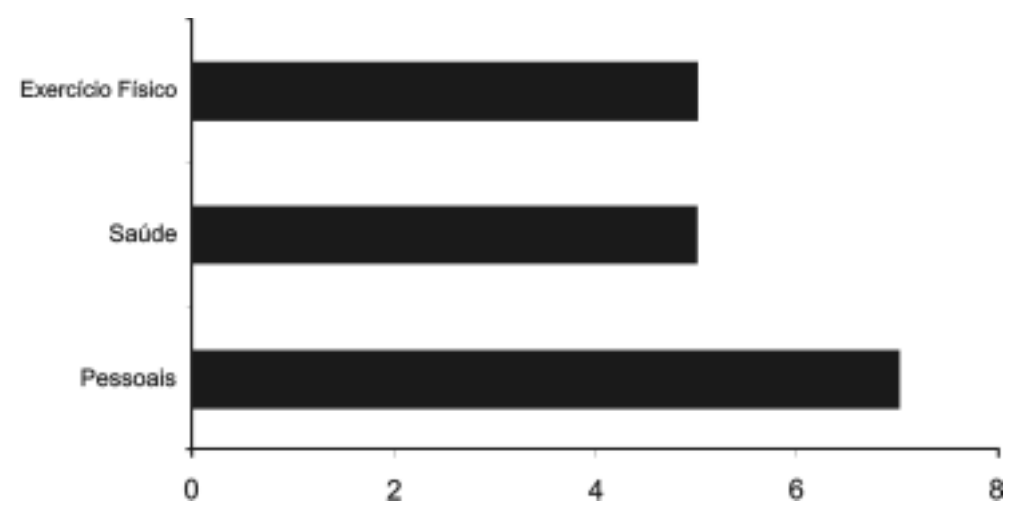

Gráfico 2. Aspectos que levaram os idosos a desistirem do programa de ginástica. Para esses aspectos uma ou mais opções foram referendadas e por isso existe discrepância quanto ao total das freqüências e a amostragem.

Estudo realizado por Santariano et al. (2000), com 2.046 pessoas acima de 55 anos, verificou que as mulheres apontaram maior número de barreiras que os homens. Entre as barreiras mais citadas estavam: a falta de companhia, a falta de interesse, a fadiga e os problemas de saúde. Os mesmos autores observaram que as razões médicas aumentaram com a idade, e que nas pessoas mais idosas ( $>75$ anos) os problemas de saúde e medo de quedas foram as barreiras mais citadas. Já Mazo (2003) cita: o estado de saúde; o medo de quedas e das suas conseqüências; a falta de motivação ou de "força de vontade". Em outro estudo, Dergance et al. (2003), avaliaram a percepção de idosos sedentários de diferentes etnias (mexicano-americanos e europeu-americanos), e observaram que a falta de interesse, disciplina, companhia, conhecimento e não gostar de fazer atividades físicas foram as barreiras que apresentaram diferença significativa entre as etnias.

No presente estudo, dos idosos que foram indagados sobre as aulas (horário, local, músicas, material, professora, exercícios), 92,3\% afirmaram gostar muito das atividades desenvolvidas, sendo que

Movimento, Porto Alegre, v. 14, n. 01, p. 225-239, janeiro/abril de 2008. 
deste percentual, 30,8\% afirmaram gostar, embora não pudessem realizar alguns exercícios, ou a aula era muito forçada ou as mudanças de professores não lhes agradavam. Neste ponto, cabe ressaltar que a atuação do professor e a criação de vínculo com os alunos é um aspecto essencial para a manutenção dos idosos em programas de exercício físico. No estudo de Freitas et al. (2007) fatores como receber incentivos e receber a atenção do professor foram apontados por $62,5 \%$ e $57,5 \%$ respectivamente, como sendo causas importantíssimas para se manterem no programa. Dos entrevistados, no presente estudo, somente uma idosa afirmou não gostar das aulas, salientando que a aula era fraca para o seu ritmo.

Em relação à sensação de bem-estar 84,6\% afirmaram sentir-se bem quando praticavam exercício físico; e destes, $15,4 \%$ sentiam-se bem num primeiro momento, mas depois sentiam dores. O restante da amostra $(15,4 \%)$ assegurou não se sentir bem com as aulas de ginástica. A importância da sensação de bem-estar propiciada pelo exercício físico para a manutenção de idosos em programas foi destacada por Freitas et al. (2007), em estudo no qual 74,2\% dos idosos entrevistados indicaram-na como sendo importante.

Entretanto, mesmo verificando problemas decorrentes do exercício físico propriamente dito e problemas de saúde, constatou-se que $84,6 \%$ têm vontade de voltar para o grupo, acreditando que seja possível sua volta, no entanto para isso, algumas situações têm que ser modificadas e muitas não dependem exclusivamente de suas ações como: melhora da saúde do cônjuge, diminuição da freqüência semanal do programa e outros aspectos que dependem das suas próprias ações como: recuperação de cirurgia e aceitação da finitude do esposo e/ou filhos.

\section{CONSIDERAÇÕES FINAIS E RECOMENDAÇÕES}

Concluiu-se que os principais motivos para adesão ao programa foram aspectos vinculados aos relacionamentos sociais, como por exemplo, convite de amigos e/ou familiares, sendo este mais

Movimento, Porto Alegre, v. 14, n. 01, p. 225-239, janeiro/abril de 2008. 
significativo que a indicação médica, apesar de ser um fator chave para a adesão de idosos a programas de exercícios físicos.

Dentre os motivos de desistência, as principais causas observadas vinculam-se a motivos pessoais, destacando-se problemas de saúde e/ou morte do cônjuge ou de demais familiares. As outras causas que levaram os idosos a desistirem do programa foram motivos de saúde e inadequação do exercício físico às suas limitações e potencialidades. Destacando-se o exercício físico propriamente dito, este fato merece atenção dos profissionais envolvidos, em especial, o profissional de Educação Física, o qual compete a intervenção por meio do exercício físico, e o devido reconhecimento das atividades a serem desenvolvidas de acordo com as necessidades com a população em questão.

Pesquisas que avaliam aos fatores de influência da prática de atividade física regular, ou sejam, os fatores que podem influenciar mais expressivamente a opção por dado comportamento, como os facilitadores e as barreiras, são fundamentais, pois este conhecimento permite estabelecer e propor programas de intervenções como meio de promoção da atividade física, que detectem, ultrapassem as barreiras e proporcionem a manutenção em níveis adequados. 
Influential factors in the desistance of aged people in a physical exercise program

Abstract: This study aimed to investigate the influential factors in the adherence and desistance of aged people in a physical exercise program implanted in Local Health Units (ULS) of Florianopolis, SC. To reach this, 13 elderly, with mean age of 71,08 $(S D=5,75)$, were interviewed by a semi-structured interview applied by telephone. It was concluded that the main adherence motive to the program was relationships aspects (friends and/or families invitation) and the mostly frequent desistance motives were personal causes (spouse's health problems, death of the spouse or other relatives). Keywords: Exercise. Adherence. Patient dropouts. Aged.

Factores influyentes en la renuncia de ancianos en un programa del ejercicio físico

Resumen : Este estudio viso a investigar los factores influyentes en la aderencia e renuncia de ancianos en un programa de ejercicio físico implantado en las Unidades del Local de Salud (ULS)de Florianópolis, SC. Para eso, se entrevistó 13 ancianos, con edad media de 71,08 ( $\mathrm{DP}=5,75)$, por medio de una entrevista semi-estructurada aplicada vía teléfono. Se concluyó que el principal motivo de aderencia a lo programa fue lo aspecto de las relaciones personales (convites de amigos y/o familiares), ya los motivos de renuncia más frecuentes fueran razones personales (problemas de salud del conjugue, muerte del mismo o de los demás familiares).

Palabras-clave: Ejercicio. Adherencia. Desistência del paciente. Anciano.

\section{REFERÊNCIAS}

BANDURA, A. Self-efficacy: toward a unifying theory of behavioral change. Psychological Review, Washington, v. 84, p. 191-215, 1997.

BIRD, S.P; TARPENNING, K.M; MARINO F.E.. Designing resistance training programmes to enhance muscular fitness: a review of the acute programme variables. Sports Med, Auckland, v. 35, n.10, p. 841-851, 2005.

Movimento, Porto Alegre, v. 14, n. 01, p. 225-239, janeiro/abril de 2008. 
BRASIL. MINISTÉRIO DA SAÚDE. Vigitel Brasil 2006: vigilância de fatores de risco e proteção para doenças crônicas por inquérito telefônico. Brasília, 2007.

BURTON, L.C.; SHAPIRO S.; GERMAN, P.S. Determinants of physical activity initiation and maintenance among community-dwelling older persons. Preventive Medicine, New Orleans, v. 29, p. 422-430, 1999.

CALFAS, K.J.; LONG, B.J.; SALLIS, J.F.; WOOTEN, W.J.; PRATT, M.; PATRICK, K.A Controlled Trial of Physician Counseling to Promote the Adoption of Physical Activity. Preventive Medicine, New Orleans, v. 25, n. 3, p. 225-233, 1996.

CHAO, D.; FOY, C.G.; FARMER, D. Exercise adherence among older adults: challenges and strategies. Controlled Clinical Trials,_v. 21, p. 212S- 217S, 2000.

COHEN-MANSFIELD, Jiska; MARX, Marcia S.; BIDDISON, James R.; GURALNIK, Jack M. Socio-environmental exercise preferences among older adults. Preventive Medicine, New Orleans, v. 38, p. 804-811, 2004.

DERGANCE, J.M. et al. Barries to and benefits of leisure time physical activity in the elderly: differences across cultures. Journal of American Geriatrics Society, v. 51, n. 6, p. 863-868, 2003.

FREITAS, C.M.S.M.; SANTIAGO, M.S.; VIANA, A.T.; LEÃO, A.C.; FREYRE, C. Aspectos motivacionais que influenciam a adesão e manutenção de idosos a programas de exercícios físicos. Revista Brasileira de Cineantropometria e Desempenho Humano, Florianópolis, v. 9, n. 1, p. 92-100, 2007.

INSTITUTO BRASILEIRO DE GEOGRAFIA E ESTATÍSTICA. Censo demográfico 2000. Rio de Janeiro, 2000.

MAGILL, R.A. Aprendizagem Motora: conceitos e aplicações. São Paulo: Edgard Blücher. 1984.

MAZO, Giovana Z. Atividade Física e Qualidade de Vida de Mulheres Idosas. 2003. 218 f. Tese (Doutorado). Faculdade de Ciências do Desporto e de Educação Física - Universidade do Porto, Porto, 2003.

MICHELI, R.S. O desafio das barreiras. Portal Fórum, maio de 2007. Disponível em: <http://www.portaldoenvelhecimento.net/pforum/afv2.htm>. Acesso em: 12 maio 2007

O'NEILL, K.; REID, G. Perceived barriers to physical activity by older adults. Canadian Journal Public Health, Ottawa, v. 82, p. 392-396, 1996.

SANTARIANO, W.A.; HAIGHT, T.J.; TAGER, I.B. Reasons given by older people for limitation or avoidence of leisure time physical activity. Journal of the American Geriatrics Society, New York ,v. 48, p. 505-512, 2000.

SCHUTZER, Karen A.; GRAVES, B. Sue. Barriers and motivations to exercise in older adults. Preventive Medicine, New Orleans, v. 39, p. 1056-1061, 2004.

Movimento, Porto Alegre, v. 14, n. 01, p. 225-239, janeiro/abril de 2008. 
SHEPHARD, R.J. Envelhecimento, Atividade Física e Saúde. São Paulo: Phorte, 2003.

WARBURTON, D.E.R; NICOL, C.W.; BREDIN, S.S.D. Health Benefits of Physical Activity: The Evidence. Canadian Medical Association Journal, Ottawa, v. 176, n. 6, p. 801-809, 2006.

Movimento, Porto Alegre, v. 14, n. 01, p. 225-239, janeiro/abril de 2008. 